\title{
Photonic non-Hermitian skin effect and non-Bloch bulk-boundary correspondence
}

\author{
Xueyi Zhu, ${ }^{1}$ Huaiqiang Wang, ${ }^{2}$ Samit Kumar Gupta $\odot,{ }^{1}$ Haijun Zhang $\odot,{ }^{2,3,4}$ \\ Biye Xie, ${ }^{1, *}$ Minghui Lu $\odot,{ }^{1,3,4, \dagger}$ and Yanfeng Chen ${ }^{1,3,4}$ \\ ${ }^{1}$ Department of Materials Science and Engineering, College of Engineering and Applied Sciences, Nanjing University, Nanjing 210093, China \\ ${ }^{2}$ School of Physics, Nanjing University, Nanjing 210093, China \\ ${ }^{3}$ National Laboratory of Solid State Microstructures, Nanjing University, Nanjing 210093, China \\ ${ }^{4}$ Collaborative Innovation Center of Advanced Microstructures, Nanjing University, Nanjing 210093, China
}

(Received 12 November 2019; accepted 12 February 2020; published 9 March 2020)

\begin{abstract}
In the area of non-Hermitian physics, there has been increasing research interest in photonics. Recently, this interest has expanded to topological systems in which symmetry and topology intertwine with non-Hermiticity, giving rise to many intriguing physical effects. One of the major tasks in exploring topological systems is unveiling the bulk-boundary correspondence in the presence of non-Hermiticity. Several proposals have been put forward in this vein, including non-Bloch bulk-boundary correspondence and the non-Hermitian skin effect. However, its practical realization has remained elusive thus far. In this paper, we demonstrate a feasible design of a one-dimensional non-Hermitian Su-Schrieffer-Heeger model based on photonic coupled resonant optical waveguides (CROWs). We show that non-Hermitian asymmetric coupling can be realized by the judicious design of optical gain and loss elements into unidirectional coupling link rings. The phase transition points of a technically achievable CROW open chain are different from those of the periodic boundary, thus revealing the non-Bloch bulk-boundary correspondence. Moreover, the field distribution is found to be exponentially localized at the ends of an open-boundary chain, which demonstrates the non-Hermitian skin effect. Our results underpin the fundamental importance as well as potential applications in various optical devices such as optical couplers, beam splitters, lasers, optical trapping, etc.
\end{abstract}

DOI: 10.1103/PhysRevResearch.2.013280

\section{INTRODUCTION}

The non-Hermitian Hamiltonian associated with open systems [1-4] and gain/loss media [5-7] has inspired significant research activities in many areas of physics and engineering [8-13]. A unique property of non-Hermitian systems is the existence of exceptional points (EPs) [14-19] corresponding to the coalescence of two or more eigenstates, which make the non-Hermitian Hamiltonian nondiagonalizable. Recently, there has been growing interest in the topological properties of non-Hermitian EPs, such as Weyl exceptional rings [20-22], bulk Fermi arcs [23], and half-integer topological charges [23]. Another remarkable phenomenon of non-Hermitian systems is the non-Hermitian skin effect, meaning that all eigenstates are localized at the boundary. As a consequence, the conventional bulk-boundary correspondence breaks down in non-Hermitian systems [24-36].

Due to the equivalence between the Schrödinger equation in quantum mechanics and the wave equation in optics, classical optical systems provide a feasible way to experimentally

\footnotetext{
*biye@nju.edu.cn

†luminghui@nju.edu.cn

Published by the American Physical Society under the terms of the Creative Commons Attribution 4.0 International license. Further distribution of this work must maintain attribution to the author(s) and the published article's title, journal citation, and DOI.
}

study the fundamentals of non-Hermitian topological properties [5,6,16-19,37-39]. In optics, the non-Hermitian potential can be efficiently constructed by engineering loss and gain modulations in a complex refractive index domain, and a large number of interesting optical phenomena and devices can be achieved in non-Hermitian optical systems, such as unidirectional reflection [17], enhanced detection [40], a PT laser $[18,19]$, and an absorber [39]. It is worth noting that coupled resonant optical waveguides (CROWs) have become an effective photonic platform for studying optical non-Hermitian topological effects [41-44]. The unidirectional coupling of the CROW structure can introduce an effective gauge field into the photonic system without time-dependent modulation or magneto-optical materials. A number of photonic nonHermitian phenomena have been realized in CROW systems, such as a topologically protected laser [43,45-48], reconfigurable light steering [49], unidirectional transmission [41,42], topological quantum devices [50,51], flat band dispersion $[44,52]$, and so on. It is worth noting that CROWs can implement asymmetric hopping with auxiliary link rings [53-55], which results in non-Hermitian transparency and one-way transport [56]. However, the non-Hermitian skin effect and bulk-boundary correspondence have not been carefully studied in a non-Hermitian CROW structure.

In this paper, we report a feasible design of a onedimensional (1D) non-Hermitian Su-Schrieffer-Heeger (SSH) model [57-60], which is a one-dimensional tight-binding model whose topological features arise from alternating offdiagonal tunneling strengths, based on the photonic CROW. 
We show that the non-Hermitian asymmetric coupling can be obtained by inserting an optical gain and loss medium into the link rings. In the simulation, the phase diagram of the CROW open-chain is different from that of the periodic boundary condition, and the non-Bloch bulk-boundary correspondence is presented, showing consistency with a theoretical model. In addition, the non-Hermitian skin effect is demonstrated by the complete localization of field distributions at the end of an open-boundary chain. The photonic non-Hermitian skin effect may provide a mechanism to strongly localize the light at the boundary and pave the way for achieving broadband lower-dimensional light trapping. Not only theoretical models, but also CMOS-compatible feasible optical designs were highlighted. Therefore, our design and results are more applicable and have the potential to be used in combination with silicon-based photonics, which may provide a feasible way for light manipulation and the design of optical devices such as optical couplers, beam splitters, lasers, and so on.

\section{THEORETICAL MODEL AND STRUCTURE DESIGN}

A tight-binding model is employed to describe the nonHermitian SSH model [28-32], which is described by the following Hamiltonian:

$$
\begin{aligned}
H(k)= & \left(\begin{array}{cc}
0 & t_{1}-\frac{\gamma}{2}+t_{2} e^{i k} \\
t_{1}+\frac{\gamma}{2}+t_{2} e^{-i k} & 0
\end{array}\right)=d_{x} \sigma_{x} \\
& +\left(d_{y}+i \frac{\gamma}{2}\right) \sigma_{y},
\end{aligned}
$$

where $d_{x}=t_{1}+t_{2} \cos (k), d_{y}=t_{2} \sin (k)$, and $\sigma_{x, y}$ are the Pauli matrices. $\gamma$ is a real number that represents the non-Hermitian coupling, which can be realized by optical gain/loss media. Here " $t$ " represents the coupling strength between two adjacent rings. The definition of $t_{1}, t_{2}$ refers to the coupling constant of two site rings within and between the unit cell. This is consistent with the coupling constant in the traditional SSH model. The unit cell has periodic boundary conditions and translational symmetry. When translating the lattice vector, wave functions only increase the Bloch phase factor $e^{i k}$. Since $t_{1}$ characterizes the coupling constant within the unit cell, no Bloch phase will occur. Gain/loss modulations are only introduced between two site rings in the unit cell, so the non-Hermitian term is only reflected in the coupling constant $t_{1}$. In contrast, there is no non-Hermitian modulation on the interunit cell coupling $t_{2}$. To introduce the asymmetric coupling into the photonic system, we design a 1D CROW photonic SSH model, which is shown in Fig. 1(a), wherein two ring waveguides with inner diameter $R_{s}=1.653 \mu \mathrm{m}$ are located. To simplify the amount of calculation, we simulate a 2D model in the full-wave simulation. Another set of boundary conditions of the 3D model in the z-direction only affects the mode parameters of the waveguide, that is, the "artificial atom" itself, but does not affect the periodic modulation of the optical lattice. Each site ring resonator is connected with its nearest-neighbor site-ring resonator via two coupling waveguides with an inner diameter $R_{l}=1.6 \mu \mathrm{m}$, which is denoted as "link rings." The whispering gallery modes (WGMs) between adjacent rings have unidirectional coupling. For example, the clockwise mode in the site ring can
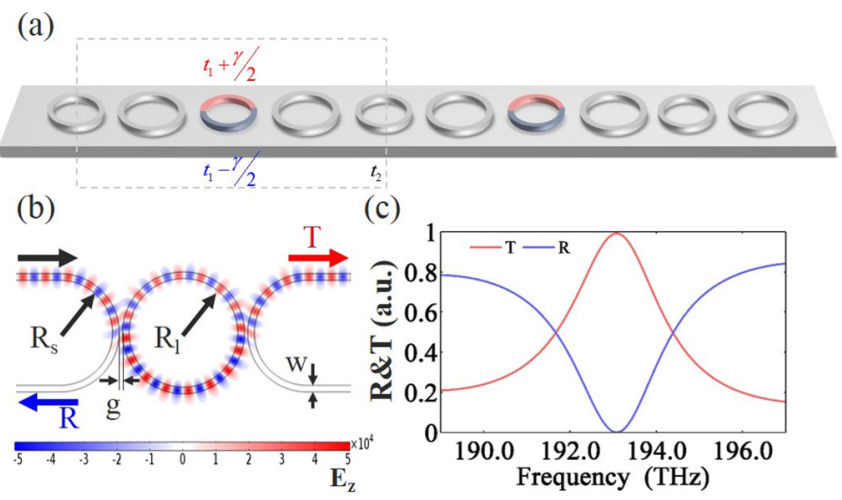

(c)

FIG. 1. (a) A schematic diagram of the CROW SSH model. The gain and loss mediums are shown as red and blue semicircles, respectively. There are two site rings with an inner diameter $R_{s}=1.653 \mu \mathrm{m}$ and two link rings with an inner diameter $R_{l}=1.6 \mu \mathrm{m}$ in the unit cell. All ring waveguides have refractive index $n=3$ and width $w=$ $0.2 \mu \mathrm{m}$. The small gap between every two resonators is $g=0.05 \mu \mathrm{m}$. (b),(c) The size parameters and the transmission/reflection spectra of ring resonators, respectively.

only excite the counterclockwise mode in the link ring. Thus, when the clockwise mode of the site ring is coupled from left to right, it passes through the lower semicircle of the link ring, and when coupled from right to left, it passes through the upper semicircle. In our design, the upper half-circle of the link ring 3 [shown in red in Fig. 1(a)] is inserted by optical gain material and the lower half contains loss. Combined with the CROW SSH model described above, we obtain a nonHermitian CROW SSH model with left and right asymmetric coupling strengths. We choose to distinguish the size of two kinds of rings to make sure that the link rings can be regarded as a coupling term between the site ring resonators. By tuning the small gap " $g$ " as shown in Figs. 1(a) and 1(b), the coupling strength between adjacent rings can be directly controlled and thus the entire CROW structure constitutes the configuration of the non-Hermitian SSH model.

In the SSH model, we often pay attention to the alternative coupling strength between adjacent atoms. With the changing of coupling strength, there is also a chance for a topology phase transition, which results in topologically protected edge states. In our design, optical resonant waveguides act as artificial atoms. The alternative coupling strength between waveguides, which can be tuned by sweeping the distance between two adjacent waveguides, also causes topological phase transitions.

Considering the actual CMOS process accuracy, we designed the gap between the site ring and the link ring $\mathrm{g}=0.05 \mu \mathrm{m}$. Increasing the gap decreases the coupling strength. Conversely, decreasing the gap increases the coupling strength. In theoretical models, the coupling constant is often directly selected for calculation. However, in an optical design, the physical quantity that can be directly controlled is the size of the gap between adjacent rings. Therefore, we choose to directly change the value of the gap to achieve the topological phase transition of the system. Since two site rings will be close to each other or away from each other in the unit cell, the distance between the site rings and the link rings will change. We define the position drift as $\delta$ and the unit is 
(a)

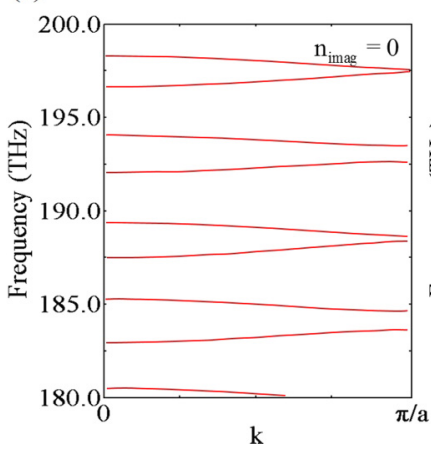

(b)

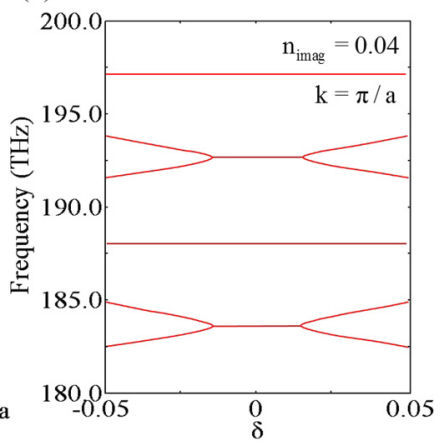

FIG. 2. (a) Schematic diagram of the energy band with no gain/loss modulation. (b) Schematic diagram of the energy band at the border of the first Brillouin zone. There are two exceptional points as the gap shift $\delta$ changes. $n_{\text {imag }}$ represents the imaginary refractive index in the full-wave simulation.

$\mu \mathrm{m}$. When $\delta$ is negative, it means that the site rings are far away from each other in the unit cell. When $\delta$ is positive, it means that the site rings are close to each other in the unit cell. Thus, the gap between the site ring and the link ring is $\mathrm{g}=0.05 \mu \mathrm{m} \pm \delta$.

\section{SIMULATION RESULTS}

As we can see in Fig. 1(c), the resonant frequency of two site rings is designed in the optical telecommunication regime from 190.0 to $196.0 \mathrm{THz}$, and the transmission rate of the ring resonator exceeds $20 \%$. The $Q$ factor of the resonant ring is so high that energy cannot be completely coupled out effectively; only a small part will be localized in the ring, which means that the field is still distinctly distributed in the upper semicircle of the link ring. It is worth noting that there is no coupling between clockwise mode and counterclockwise mode in the link ring. For the energy incident from the upperleft incident port, there is only one coupling channel that can couple with the right out port. Therefore, the small part of the energy that is localized in the ring will not affect the Hamiltonian.

Through full-wave simulation, we obtain the energy band of the SSH model based on the photonic CROW structure as shown in Fig. 2(a). In the simulation, we calculated the eigenstates in a unit cell that contains four resonances. In the resonance frequency region of the site ring, a photonic band gap formed emerges at the border of the Brillouin zone due to the alternate coupling coefficient. It is worth mentioning that the optical ring waveguide supports both clockwise and counterclockwise WGMs, and the two modes are completely degenerate. The coupling between clockwise and counterclockwise modes within an isolated ring is forbidden by rotation and time-reversal symmetries $[41,42,44]$. Without loss of generality, here we only consider one of the two WGMs.

The energy bands of the Hamiltonian are obtained by diagonalizing the above matrix, and the energy gap closes at the EPs $t_{1}=t_{2} \pm \frac{\gamma}{2}(k=\pi)$ under periodic boundary conditions. By changing the spacing between the rings to control the coupling strength, we can obtain the energy band evolution of the high symmetric point in the Brillouin zone in full- wave simulation. In our system, the phase transition point specifically refers to the critical state when the band gap of the system's energy band changes from closed to open or from open to closed and the topological phase changes. By changing the coupling coefficient ( $\delta$ is related to the coupling coefficient), we successfully obtain the phase transition point at the $k$ point, as shown in Fig. 2(b). Two EPs corresponding to the theoretical model can be obtained. For the traditional SSH model, there is a phase transition point. However, the imaginary part of the ring resonant we choose in Fig. 2(b) is $0.04\left(n_{\text {imag }}\right)$, so two phase transition points appear and the CROW system is gapless not only on the phase transition point but also between them.

In our non-Hermitian CROW SSH model, the open boundary case is noticeably different from that of the periodic boundary. For open boundaries, there is no translation symmetry in space. Therefore, to describe the Hamiltonian of the system, a large matrix with the same matrix dimension as the number of long-chain site rings must be established to describe the Hamiltonian of the system. If there are $N$ site rings in the system, the matrix is $\mathrm{N}$-dimensional. Such an open-chain system can be described by the Hamiltonian below:

$$
\mathrm{H}_{N \times N}=\left(\begin{array}{ccccc}
0 & t_{1}-\frac{\gamma}{2} & 0 & \ldots & 0 \\
t_{1}+\frac{\gamma}{2} & 0 & t_{2} & \ldots & 0 \\
0 & t_{2} & 0 & \ldots & 0 \\
\vdots & \vdots & \vdots & \vdots & \vdots \\
0 & 0 & 0 & t_{1}+\frac{\gamma}{2} & 0
\end{array}\right) .
$$

The phase boundary position of the CROW open chain is given by $t_{1}=\sqrt{t_{2}^{2}+\left(\frac{\gamma}{2}\right)^{2}}$ when $\left|t_{1}\right|>|\gamma / 2|$ [32], which is drastically different from that of the periodic boundary $t_{1}=t_{2} \pm \frac{\gamma}{2}(k=\pi)$ indicating the non-Bloch bulk-boundary correspondence. In the Hermitian case, the boundary condition has little effect on the eigenstate, so the Hermitian topological system preserves bulk-boundary correspondence. However, in the non-Hermitian case, the boundary condition has a nontrivial influence on the eigenstate. The eigenstates cannot be superimposed by Bloch wave vectors, and the field distribution has a non-Hermitian skin effect, resulting in the non-Bloch bulk-boundary correspondence.

We have successfully achieved similar results through fullwave simulation. To get the phase diagrams (Fig. 3) and the field distributions (Fig. 4), we use 20 unit cells in the full-wave simulation, each of which contains two site rings and two link rings. All unit cells are arranged along the $x$-axis direction. It is worth noting that $N$ represents the number of site rings. The link ring only provides coupling coefficient modulation to achieve asymmetric coupling. We set periodic boundary conditions and scattering boundary conditions at the left and right boundaries, so that we can calculate the system eigenvalues under the periodic and open boundary, respectively. The phase transition point under the periodic boundary $(\delta=00.11)$ is different from that under the open boundary $(\delta=0.02)$. The phase transition point under the periodic boundary [the bottom panel of Fig. 3(a)] is different from that under the open boundary [the top panel of Fig. 3(a)]. The edge state starts to appear at the phase transition point 


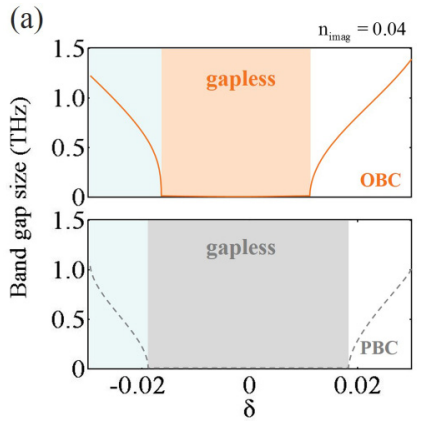

(b)

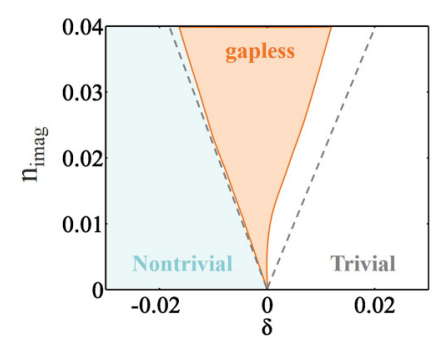

FIG. 3. (a) Phase diagram based on the spectra of the CROW SSH model. The top and bottom panels correspond to the open boundary condition (OBC) and the periodic boundary condition (PBC), respectively. Edge states can be found in the entire blue shadow area, indicating that the region is nontrivial. (b) Topological phase diagram under different gain/loss modulations. The yellow solid line and gray dashed line indicate the position of the phase transition point under the open boundary and the periodic boundary, respectively.

of the open boundary (blue shadow area), indicating that the region is nontrivial [61].

The Hamiltonian of the CROW SSH model can be rewritten under the non-Hermitian open boundary condition by reconstructing the basis vector. The real-space eigenequation can be rewritten as $\bar{H}|\bar{\psi}\rangle=E|\bar{\psi}\rangle$, with $|\bar{\psi}\rangle=S^{-1}|\psi\rangle$. We can choose the trial solution $S$ in this similarity transformation according to the characteristics of the non-Hermitian wave function. The $S$ is taken to be a diagonal matrix whose diagonal elements are $\left\{1, r, r, r^{2}, r^{2}, \ldots, r^{L-1}, r^{L-1}, r^{L}\right\}$. Then $t_{1} \pm \frac{\gamma}{2}$ terms can be replaced by $r^{ \pm 1}\left(t_{1} \pm \frac{\gamma}{2}\right)$ in $\bar{H}$. The non-Hermitian variation of the wave function is taken into account. The diagonal element $r$ can be solved by boundary conditions. Now the trial solution $r$ can be chosen as $r=\sqrt{\left|\left(t_{1}-\gamma / 2\right)\right| /\left|\left(t_{1}+\gamma / 2\right)\right|}$. Thus the Hamiltonian of the CROW open-chain can be represented as

$$
\bar{H}_{N \times N}=\left(\begin{array}{ccccc}
0 & \left(t_{1}-\frac{\gamma}{2}\right) / r & 0 & \cdots & 0 \\
\left(t_{1}+\frac{\gamma}{2}\right) r & 0 & t_{2} & \ldots & 0 \\
0 & t_{2} & 0 & \ldots & 0 \\
\vdots & \vdots & \vdots & \vdots & \vdots \\
0 & 0 & 0 & \left(t_{1}+\frac{\gamma}{2}\right) r & 0
\end{array}\right) .
$$

The form is consistent with the standard $\mathrm{SSH}$ model: $\bar{H}(k)=\left(\overline{t_{1}}+\overline{t_{2}} \cos k\right) \sigma_{x}+\overline{t_{2}} \sin k \sigma_{y}$, where $\overline{t_{1}}=$

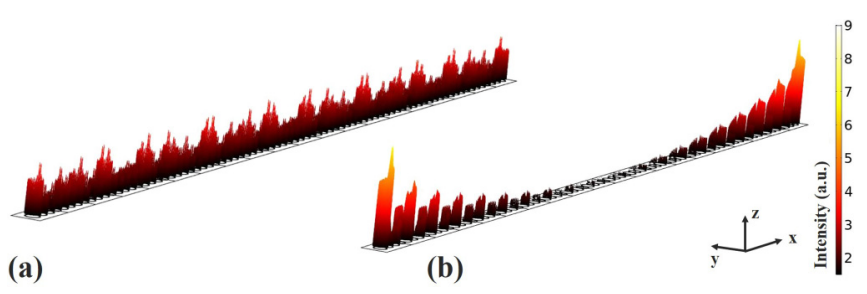

FIG. 4. Field distribution of bulk states, which shows a comparison of the Hermitian bulk state (a) and the obvious non-Hermitian skin effect (b). $\sqrt{\left(t_{1}-\gamma / 2\right) /\left(t_{1}+\gamma / 2\right)}$ and $\overline{t_{2}}=t_{2}$. The positions of the phase transition points are $\overline{t_{1}}=\overline{t_{2}}$, which means $t_{1}=\sqrt{t_{2}^{2}+(\gamma / 2)^{2}}$ [61]. This is consistent with the theoretical calculation results.

In the full-wave simulation, we obtain the topological phase diagram, which presents the phase transition points under different gain/loss modulations, as shown in Fig. 3(b). Under open boundary conditions, the phase transition point corresponds to the position where the edge state begins to appear, and edge states can be found in the entire blue shadow area, indicating that the region is nontrivial. For the entire eigenequation, there is only a mathematical form of change in the Hamiltonian basis vector. However, due to the introduction of gain/loss modulation, the original basis vectors cannot describe the behavior of the system, nor can they explain the occurrence of bulk-boundary correspondence. However, the bulk-boundary correspondence can be explained with the basis transformation. This is also confirmed in our simulation results.

In the non-Hermitian SSH model, the wave function has amplification/attenuation and phase changes during propagation, which is manifested by the non-Hermitian skin effect of the field distribution. In other words, all the bulk state fields are concentrated at the boundary, analogous to the non-Hermitian skin effect of electromagnetic waves on the metal surface. For the general bulk state [Fig. 4(a)], its field distribution is evenly distributed throughout the system. Due to the differences in field excitations, the field distribution in our design has certain differences at different frequencies. However, all the bulk states in our design have above $60 \%$ energy concentrated in the three rings of the open-chain border, thus confirming the existence of the non-Hermitian skin effect in the non-Hermitian CROW SSH model [Fig. 4(b)]. It is worth noting that the non-Hermitian optical modulation experienced by clockwise and counterclockwise WGMs is opposite, resulting in the fact that they concentrate at different boundaries. So, the bulk field distribution is concentrated on both sides of the open-chain boundary.

\section{CONCLUSION}

In conclusion, we have designed a non-Hermitian 1D SSH model based on the photonic CROW structure. Different from the previous non-Hermitian SSH model where the nonHermitian modulation is located at the site position and corresponds to the diagonal term of the Hamiltonian, our CROW SSH model possesses the link ring modulation corresponding to the off-diagonal terms of the Hamiltonian. To realize a synthetic imaginary asymmetric coupling, the link ring provides amplification in the upper half perimeter with single-pass amplification and balanced loss in the lower half perimeter with single-pass attenuation. The topological phase transition point of the CROW open chain is different from that of the periodic boundary and the present non-Bloch bulk-boundary correspondence. In the non-Hermitian SSH model, the field distribution displays a complete localization at the end of an open-boundary chain, indicating the existence of the non-Hermitian skin effect. The 1D non-Hermitian CROW SSH model provides an efficient platform for designing integrated topological photonic devices and topological quantum optical devices. The non-Hermitian skin effect may 
be suggestive of potential application prospects and research values in optical devices such as optical couplers, beam splitters, lasers, and so on.

\section{ACKNOWLEDGMENTS}

The authors acknowledge the support from the National Key R\&D Program of China (NKRDP) (Grants
No. 2018YFA0306200 and No. 2017YFA0303702), National Natural Science Foundation of China (NSFC) (Grants No. 11625418, No. 51732006, and No. 11674165), the Fok Ying-Tong Education Foundation of China (Grant No. 161006), China Postdoctoral Science Foundation Funded Project (Project No. 2019M661784), and the Fundamental Research Funds for the Central Universities (Grant No. 020414380038).
[1] B. Ostahie, M. Niţa, and A. Aldea, Non-Hermitian approach of edge states and quantum transport in a magnetic field, Phys. Rev. B 94, 195431 (2016).

[2] T. E. Lee and C.-K. Chan, Heralded Magnetism in NonHermitian Atomic Systems, Phys. Rev. X 4, 041001 (2014).

[3] S. Diehl, E. Rico, M. A. Baranov, and P. Zoller, Topology by dissipation in atomic quantum wires, Nat. Phys. 7, 971 (2011).

[4] M. S. Rudner and L. S. Levitov, Topological Transition in a Non-Hermitian Quantum Walk, Phys. Rev. Lett. 102, 065703 (2009).

[5] A. Regensburger, C. Bersch, M.-A. Miri, G. Onishchukov, D. N. Christodoulides, and U. Peschel, Parity-time synthetic photonic lattices, Nature (London) 488, 167 (2012).

[6] L. Feng, R. El-Ganainy, and L. Ge, Non-Hermitian photonics based on parity-time symmetry, Nat. Photon. 11, 752 (2017).

[7] C. M. Bender and S. Boettcher, Real Spectra in Non-Hermitian Hamiltonians Having PT Symmetry, Phys. Rev. Lett. 80, 5243 (1998).

[8] D. Leykam, K. Y. Bliokh, C. Huang, Y. D. Chong, and F. Nori, Edge Modes, Degeneracies, and Topological Numbers in Non-Hermitian Systems, Phys. Rev. Lett. 118, 040401 (2017).

[9] T. Liu, Y. R. Zhang, Q. Ai, Z. Gong, K. Kawabata, M. Ueda, and F. Nori, Second-Order Topological Phases in Non-Hermitian Systems, Phys. Rev. Lett. 122, 076801 (2019).

[10] S. Malzard, C. Poli, and H. Schomerus, Topologically Protected Defect States in Open Photonic Systems with Non-Hermitian Charge-Conjugation and Parity-Time Symmetry, Phys. Rev. Lett. 115, 200402 (2015).

[11] J. H. Wu, M. Artoni, and G. C. La Rocca, Non-Hermitian Degeneracies and Unidirectional Reflectionless Atomic Lattices, Phys. Rev. Lett. 113, 123004 (2014).

[12] J. M. Zeuner, M. C. Rechtsman, Y. Plotnik, Y. Lumer, S. Nolte, M. S. Rudner, M. Segev, and A. Szameit, Observation of a Topological Transition in the Bulk of a Non-Hermitian System, Phys. Rev. Lett. 115, 040402 (2015).

[13] Z. Gong, Y. Ashida, K. Kawabata, K. Takasan, S. Higashikawa, and M. Ueda, Topological Phases of Non-Hermitian Systems, Phys. Rev. X 8, 031079 (2018).

[14] W. Hu, H. Wang, P. P. Shum, and Y. D. Chong, Exceptional points in a non-Hermitian topological pump, Phys. Rev. B 95, 184306 (2017).

[15] C. Hahn, Y. Choi, J. W. Yoon, S. H. Song, C. H. Oh, and P. Berini, Observation of exceptional points in reconfigurable nonHermitian vector-field holographic lattices, Nat. Commun. 7, 12201 (2016).

[16] X.-Y. Zhu, Y.-L. Xu, Y. Zou, X.-C. Sun, C. He, M.-H. Lu, X.-P. Liu, and Y.-F. Chen, Asymmetric diffraction based on a passive parity-time grating, Appl. Phys. Lett. 109, 111101 (2016).
[17] L. Feng, Y.-L. Xu, W. S. Fegadolli, M.-H. Lu, J. E B. Oliveira, V. R. Almeida, Y.-F. Chen, and A. Scherer, Experimental demonstration of a unidirectional reflectionless parity-time metamaterial at optical frequencies, Nat. Mater. 12, 108 (2013).

[18] L. Feng, Z. J. Wong, R.-M. Ma, Y. Wang, and X. Zhang, Singlemode laser by parity-time symmetry breaking, Science 346, 972 (2014).

[19] H. Hodaei, M.-A. Miri, M. Heinrich, D. N. Christodoulides, and M. Khajavikhan, Parity-time-symmetric microring lasers, Science 346, 975 (2014).

[20] A. Cerjan, S. Huang, M. Wang, K. P. Chen, Y. Chong, and M. C. Rechtsman, Experimental realization of a Weyl exceptional ring, Nat. Photon. 13, 623 (2019).

[21] B. Zhen, C. W. Hsu, Y. Igarashi, L. Lu, I. Kaminer, A. Pick, S.-L. Chua, J. D. Joannopoulos, and M. Soljačić, Spawning rings of exceptional points out of Dirac cones, Nature (London) 525, 354 (2015).

[22] Y. Xu, S.-T. Wang, and L.-M. Duan, Weyl Exceptional Rings in a Three-Dimensional Dissipative Cold Atomic Gas, Phys. Rev. Lett. 118, 045701 (2017).

[23] H. Zhou, C. Peng, Y. Yoon, C. W. Hsu, K. A. Nelson, L. Fu, J. D. Joannopoulos, M. Soljačić, and B. Zhen, Observation of bulk Fermi arc and polarization half charge from paired exceptional points, Science 359, 1009 (2018).

[24] L. Jin and Z. Song, Bulk-boundary correspondence in a nonHermitian system in one dimension with chiral inversion symmetry, Phys. Rev. B 99, 081103 (2019).

[25] K. Kawabata, K. Shiozaki, and M. Ueda, Anomalous helical edge states in a non-Hermitian Chern insulator, Phys. Rev. B 98, 165148 (2018).

[26] V. M. Martinez Alvarez, J. E. Barrios Vargas, and L. E. F. Foa Torres, Non-Hermitian robust edge states in one dimension: Anomalous localization and eigenspace condensation at exceptional points, Phys. Rev. B 97, 121401 (2018).

[27] H. Wang, J. Ruan, and H. Zhang, Non-Hermitian nodal-line semimetals with an anomalous bulk-boundary correspondence, Phys. Rev. B 99, 075130 (2019).

[28] F. K. Kunst, E. Edvardsson, J. C. Budich, and E. J. Bergholtz, Biorthogonal Bulk-Boundary Correspondence in Non-Hermitian Systems, Phys. Rev. Lett. 121, 026808 (2018).

[29] T. E. Lee, Anomalous Edge State in a Non-Hermitian Lattice, Phys. Rev. Lett. 116, 133903 (2016).

[30] H. Shen, B. Zhen, and L. Fu, Topological Band Theory for NonHermitian Hamiltonians, Phys. Rev. Lett. 120, 146402 (2018).

[31] S. Yao, F. Song, and Z. Wang, Non-Hermitian Chern Bands, Phys. Rev. Lett. 121, 136802 (2018).

[32] S. Yao and Z. Wang, Edge States and Topological Invariants of Non-Hermitian Systems, Phys. Rev. Lett. 121, 086803 (2018). 
[33] K. Yokomizo and S. Murakami, Non-Bloch Band Theory of Non-Hermitian Systems, Phys. Rev. Lett. 123, 066404 (2019).

[34] C. H. Lee and R. Thomale, Anatomy of skin modes and topology in non-Hermitian systems, Phys. Rev. B 99, 201103 (2019).

[35] F. Song, S. Yao, and Z. Wang, Non-Hermitian Skin Effect and Chiral Damping in Open Quantum Systems, Phys. Rev. Lett. 123, 170401 (2019).

[36] L. Xiao, T. Deng, K. Wang, G. Zhu, Z. Wang, W. Yi, and P. Xue, Observation of non-Hermitian bulk-boundary correspondence in quantum dynamics, arXiv:1907.12566.

[37] Y. C. Hu and T. L. Hughes, Absence of topological insulator phases in non-Hermitian PT-symmetric Hamiltonians, Phys. Rev. B 84, 153101 (2011).

[38] S. Weimann, M. Kremer, Y. Plotnik, Y. Lumer, S. Nolte, K. G. Makris, M. Segev, M. C. Rechtsman, and A. Szameit, Topologically protected bound states in photonic parity-time-symmetric crystals, Nat. Mater. 16, 433 (2017).

[39] Z. J. Wong, Y.-L. Xu, J. Kim, K. O’Brien, Y. Wang, L. Feng, and $X$. Zhang, Lasing and anti-lasing in a single cavity, Nat. Photon. 10, 796 (2016).

[40] W. Chen, Ş. K. Özdemir, G. Zhao, J. Wiersig, and L. Yang, Exceptional points enhance sensing in an optical microcavity, Nature (London) 548, 192 (2017).

[41] M. Hafezi, E. A. Demler, M. D. Lukin, and J. M. Taylor, Robust optical delay lines with topological protection, Nat. Phys. 7, 907 (2011).

[42] M. Hafezi, S. Mittal, J. Fan, A. Migdall, and J. M. Taylor, Imaging topological edge states in silicon photonics, Nat. Photon. 7, 1001 (2013).

[43] H. Zhao, P. Miao, M. H. Teimourpour, S. Malzard, R. ElGanainy, H. Schomerus, and L. Feng, Topological hybrid silicon microlasers, Nat. Commun. 9, 981 (2018).

[44] X.-Y. Zhu, S. K. Gupta, X.-C. Sun, C. He, G.-X. Li, J.-H. Jiang, X.-P. Liu, M.-H. Lu, and Y.-F. Chen, Z2 topological edge state in honeycomb lattice of coupled resonant optical waveguides with a flat band, Opt. Express 26, 24307 (2018).

[45] B. Bahari, A. Ndao, F. Vallini, A. El Amili, Y. Fainman, and B. Kanté, Nonreciprocal lasing in topological cavities of arbitrary geometries, Science 358, 636 (2017).

[46] M. A. Bandres, S. Wittek, G. Harari, M. Parto, J. Ren, M. Segev, D. N. Christodoulides, and M. Khajavikhan, Topo- logical insulator laser: Experiments, Science 359, eaar4005 (2018).

[47] G. Harari, M. A. Bandres, Y. Lumer, M. C. Rechtsman, Y. D. Chong, M. Khajavikhan, D. N. Christodoulides, and M. Segev, Topological insulator laser: Theory, Science 359, eaar4003 (2018).

[48] S. Malzard, E. Cancellieri, and H. Schomerus, Topological dynamics and excitations in lasers and condensates with saturable gain or loss, Opt. Express 26, 22506 (2018).

[49] H. Zhao, X. Qiao, T. Wu, B. Midya, S. Longhi, and L. Feng, Non-Hermitian topological light steering, Science 365, 1163 (2019).

[50] S. Barik, A. Karasahin, C. Flower, T. Cai, H. Miyake, W. DeGottardi, M. Hafezi, and E. Waks, A topological quantum optics interface, Science 359, 666 (2018).

[51] S. Mittal, E. A. Goldschmidt, and M. Hafezi, A topological source of quantum light, Nature (London) 561, 502 (2018).

[52] L. Ge, Non-Hermitian lattices with a flat band and polynomial power increase, Photon. Res. 6, A10 (2018).

[53] S. Longhi, D. Gatti, and G. Della Valle, Robust light transport in non-Hermitian photonic lattices, Sci. Rep. 5, 13376 (2015).

[54] S. Longhi, Parity-time symmetry meets photonics: A new twist in non-Hermitian optics, Europhys. Lett. 120, 64001 (2017).

[55] S. Longhi, Non-Hermitian gauged topological laser arrays, Ann. Phys. (Leipzig) 530, 1800023 (2018).

[56] S. Longhi, D. Gatti, and G. Della Valle, Non-Hermitian transparency and one-way transport in low-dimensional lattices by an imaginary gauge field, Phys. Rev. B 92, 094204 (2015).

[57] W. P. Su, J. R. Schrieffer, and A. J. Heeger, Solitons in Polyacetylene, Phys. Rev. Lett. 42, 1698 (1979).

[58] S. Ryu and Y. Hatsugai, Topological Origin of Zero-Energy Edge States in Particle-Hole Symmetric Systems, Phys. Rev. Lett. 89, 077002 (2002).

[59] E. J. Meier, F. A. An, and B. Gadway, Observation of the topological soliton state in the Su-Schrieffer-Heeger model, Nat. Commun. 7, 13986 (2016).

[60] M. Atala, M. Aidelsburger, J. T. Barreiro, D. Abanin, T. Kitagawa, E. Demler, and I. Bloch, Direct measurement of the Zak phase in topological Bloch bands, Nat. Phys. 9, 795 (2013).

[61] See Supplemental Material at http://link.aps.org/supplemental/ 10.1103/PhysRevResearch.2.013280 for details of the calculation. 